\title{
Stereoscopic evoked responses to crossed and uncrossed disparity accompanying simulated refractive error
}

\author{
R A NEILl, P DUNLOP, D B DUNLOP, B FENELON, AND C DUNLOP \\ From the University of Newcastle, NSW, Australia 2308
}

SUMMARY Evoked potentials were recorded to the occurrence of a disparate stimulus in dynamic random dot stereograms. Seven adult males, all of whom had vision which was normal or corrected to normal, participated in the experiment. Subjects viewed $100 \mathrm{~ms}$ duration stimuli which embodied 30 arc min of either crossed or uncrossed disparity under four conditions of spherical overcorrection: $-0 \cdot 25,+1 \cdot 0,+2 \cdot 0,+3 \cdot 0$ dioptres. The first condition, essentially normal refraction, yielded reliable behavioural reports of the stimulus and clear evoked potentials to both crossed and uncrossed disparity. With increasing overcorrection the behavioural reports became less reliable, and the evoked potentials were degraded for both conditions of disparity. The responses to the crossed disparity condition, however, showed significantly less degradation in both behavioural and electrophysiological measures. The implications of this finding may be that there are separate cortical subsystems for the processing of crossed and uncrossed disparity and that the former is more robust under non-ideal viewing conditions.

The human visual system processes the differences between the images formed on the spatially separated right and left retinae and uses the differences in forming a single, fused, three-dimensional image. These differences can be considered in angular terms. As a first approximation light rays travel straight from an object of interest through the nodal points of the eyes to the retinae, as illustrated in Fig. 1. Let the angle between the fixation rays be called angle $F$ and between the rays emanating from the object of interest be angles $X_{1}, X_{2}$, etc. If angle $X_{1}$ is equal to angle $F$, then the object lies on the horopter, that is, it lies in the fixation plane. The angle $X_{1}$ is greater than angle $F$, and the image of the object is said to produce a crossed disparity, because rays carried through to the horopter from the right eye pass the fixation point to the left and vice versa. The angle $X_{2}$ is less than angle $F$, producing an uncrossed disparity.

Microelectrode studies have shown that in monkeys there are separate groups of cortical neurones dedicated to the processing of crossed and uncrossed disparity. ${ }^{12}$ It is likely that such neuronal

Correspondence to Dr R A Neill. groups also exist in man. These groups are a part of a human stereoscopic visual system which serves at least two functions: (1) it allows the observer to judge precisely the position of objects, relative to some fixation point, within the visual field; (2) it aids the observer in the estimation of direction and velocity of objects which are moving within the visual field. On an intuitive level it would seem more important for an observer to judge correctly the position and speed of an object which is approaching than of one which is moving away. It is suggested, therefore, that the ability to process changes in disparity, in cases where the change is in the crossed disparity direction, will be found superior to the uncrossed disparity counterparts. It is further suggested that crossed disparity processing will be found more robust under non-ideal viewing conditions, such as may arise in the presence of a significant refractive error. The first hypothesis received strong support from a behavioural study ${ }^{3}$ in which measures of minimum presentation times for the detection of disparate stimuli showed significant crossed/uncrossed disparity differences. The hypothesis also received support from an electrophysiological study in which responses to crossed and uncrossed disparities were recorded in the upper, 


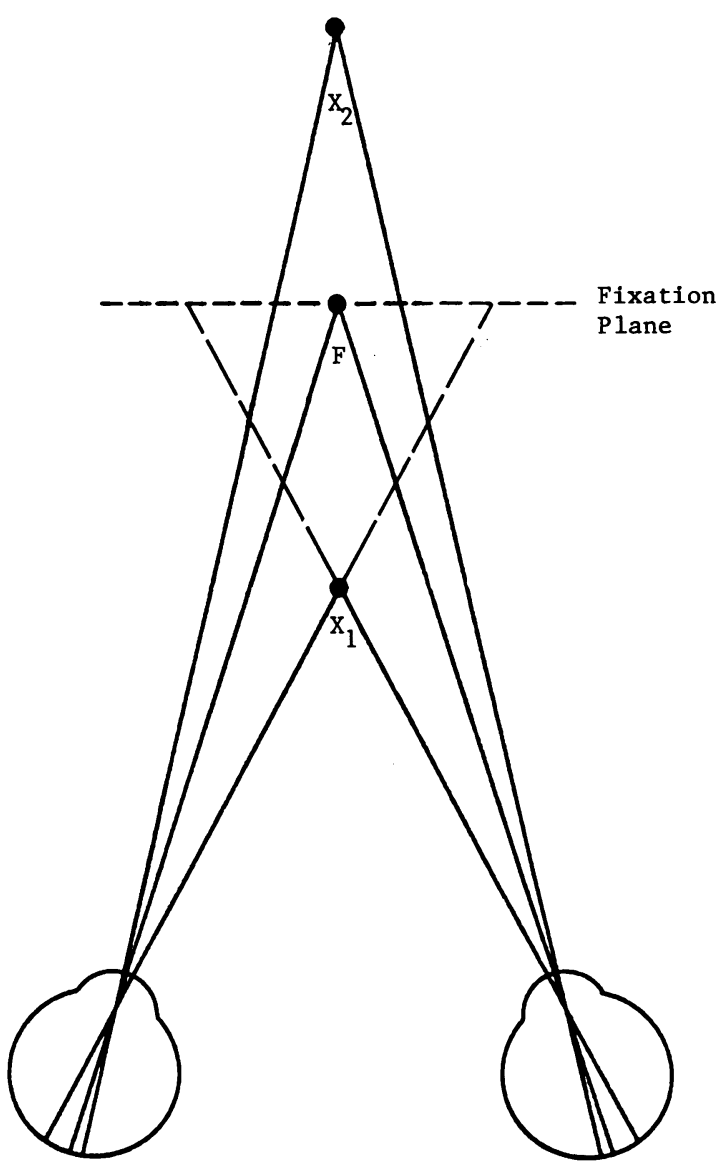

Fig. 1 Illustration of crossed and uncrossed disparity. The difference $\left(F-X_{1}\right)$ represents a crossed disparity, while $\left(F-X_{2}\right)$ represents an uncrossed disparity.

centre, and lower visual fields. ${ }^{4}$ The aim of this paper is to test the second hypothesis by investigating the electrophysiological responses to the introduction of crossed and uncrossed disparity under conditions of simulated refractive error.

\section{Material and methods}

Visual stimuli comprised dynamic random dot stereograms. These were presented in a point-to-point display mode on separate (left and right) Tektronix 602 display units (P31 phosphor). The display units were supported vertically on trolleys which were attached to the moving arms of a Clement Clarke synoptophore. ${ }^{5}$ The images of the display units were reflected into the eyepieces via front-surfaced, halfsilvered mirrors. Fixation points, subtending $0 \cdot 14^{\circ}$ and illuminated by red LEDs, were positioned centrally at the fixation distance behind the mirrors.
The random dot display, generated by a computer controlled, hardwired unit, ${ }^{6}$ comprised a $256 \times 256$ pixel square matrix subtending $10.7^{\circ}$ on the side. The disparate stimulus, when present, was located centrally. It was a square, subtending $3^{\circ}$ on the side and with a disparity of 30 arc min. By interchanging the left and right displays a crossed or uncrossed stimulus could be created. It should be noted that, unlike real space tests, there were no optical differences between the two disparity conditions. Dot presentation rate was 100000 point pairs per second.

Subjects. Seven male subjects, ages $32 \cdot 6$ (SD 7.3) years, all right handed and with normal or corrected to normal visual acuity, volunteered to participate in the experiment.

Electrophysiological responses were recorded from four scalp sites: 02,01,T6,T5 (International 10-20 System) and referenced to Fz10, a midline site $10 \mathrm{~cm}$ posterior to the nasion. Eye movements were monitored from a single channel by means of electrodes placed diagonally across the eyes. Chlorided silver electrodes were used (Burden type), and scalp impedances were kept at $3 \mathrm{kOhms}( \pm 10 \%)$ throughout the experiment.

Data were recorded at a rate of one five-channel sample every four milliseconds for a one second epoch. Low-pass filters were set at $30 \mathrm{~Hz}(3 \mathrm{~dB}$ down) and high-pass at $0.5 \mathrm{~Hz}$.

The Experiment. Subjects were screened for visual acuity with a Snellen chart. They then underwent a series of static stereopsis tests involving the Randot (Stereo Optical Co.), Neill Stereo Wedge (Clement Clarke), and synoptophore numbered shapes (Clement Clarke, slides D21,22) tests. In order to facilitate correct fusion, visual alignment was checked on the synoptophore with Mayou No. 2 simultaneous perception slides. All subjects were within normal limits on every test.

Subjects were seated in a comfortable viewing position in a light-attenuated room. The synoptophore eyepieces were initially arranged with a $-\mathbf{0 . 2 5}$ dioptre allowance for instrument accommodation. The instrument therefore had an optical viewing distance of $4 \mathrm{~m}$. The subject viewed 32 presentations each of the crossed and uncrossed disparity conditions, along with four other 32-trial conditions reported elsewhere. ${ }^{4}$ Separate averaged evoked potentials were recorded for each of these conditions. The eyepiece lenses were then replaced with pairs of lenses which were either $+1 \cdot 0,+2 \cdot 0$, or $+3 \cdot 0$ sphere,dioptres over-power. Four sets of the 32-trial averages were then recorded for each of the central crossed and uncrossed stimuli, the order being randomised. This group of trials was repeated twice more with different pairs of eyepiece lenses each time. The order of selection of lenses was randomised 
across subjects. Finally, the -0.25 dioptre lenses were replaced and three more sets of the averages recorded, along with 12 other conditions. ${ }^{4}$ Thus four sets of 32-trial averages were recorded for all conditions. The stimulus onset, comprising the abrupt introduction of disparity, occurred $100 \mathrm{~ms}$ into the recording epoch, and the duration of the disparity was $100 \mathrm{~ms}$.

A PDP 12 computer controlled the experiment, recorded and stored electrophysiological responses, and was responsible for the performance of off-line data processing.

Data analyses. For each subject and each condition the four sets of 32-trial averages were combined into 128-trial superaverages. These were then normalised across the $100 \mathrm{~ms}$ prestimulus interval. The mean amplitudes were then calculated across a 100 ms window commencing 200 ms post stimulus. The mean amplitudes were then entered into a 4 (dioptre) $\times 2$ (crossed/uncrossed) $\times 4$ (site) analysis of variance (ANOVA). In order to test the relationship between amplitude of response and degree of refractive defocusing, an amplitude versus dioptre linear regression was performed on each subject's data and the slope estimates of the regression lines were submitted to a 2 (crossed/uncrossed) $\times 4$ (site) ANOVA.

\section{Results}

BEHAVIOURAL

All subjects were able to identify the onset and orientation of the crossed stimulus for all refraction conditions, though they reported some difficulty in the +3.0 dioptre condition. All subjects, furthermore, correctly reported seeing the uncrossed stimulus in the $-0 \cdot 25$ dioptre condition, and six correctly described the +1.0 dioptre condition. Only two subjects, however, could reliably identify the presence of the uncrossed stimulus in the +2.0 dioptre condition, and no subject reported seeing this stimulus in the $+3 \cdot 0$ dioptre condition.

\section{ELECTROPHYSIOLOGICAL}

The superaveraged evoked responses for each of the conditions are included in Fig. 2 (sites 02, 01 only). Two trends are evident from the diagram and are confirmed by ANOVA. The responses to both crossed and uncrossed stimuli became smaller as the degree of simulated refractive error increased, the main effect for dioptre being significant $(F(3,18)=$ 9.93, $\mathrm{p}<0.0004)$. The responses to the uncrossed stimulus, furthermore, were smaller overall than those to the crossed stimulus (respective mean amplitudes -1.3 and -2.0 microvolts), the main effect for
Site:

01

Disparity: Crossed

$-0.25$
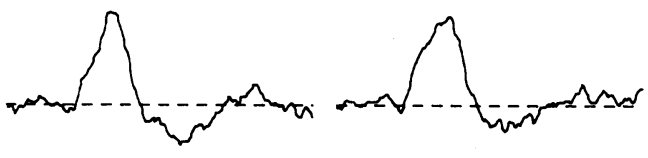

Uncrossed

Crossed
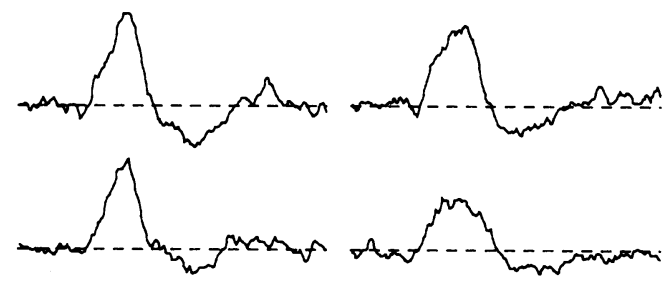

$+2.0$
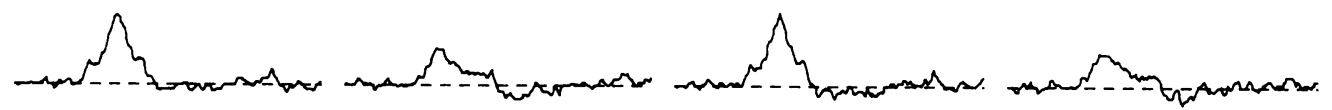

$+3.0$
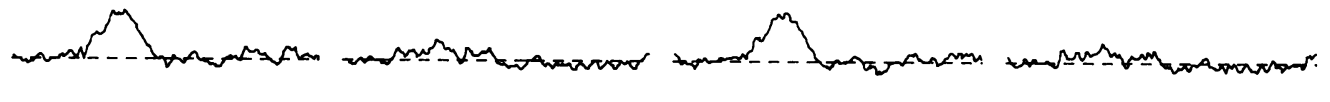

Over-

correction

(Dioptre)

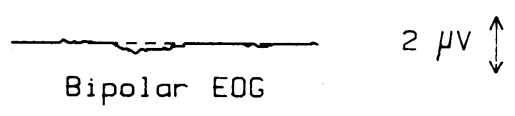

Fig. 2 Superaveraged evoked responses (sites 01 and 02, Negative up) to the crossed and uncrossed disparity across all refraction conditions. 
crossed/uncrossed also being significant $(F(1,6)=$ 26.07, $\mathrm{p}<0.003)$. The remaining main effect, site, was not significant.

Another trend evident in Fig. 2 is that the responses to the uncrossed stimulus were more heavily attenuated by the over-refraction than those to the crossed stimulus. In the normal viewing condition $(-0.25$ dioptre), the responses were of similar size (crossed $=-2 \cdot 6$, uncrossed $=-2 \cdot 4$ microvolt). For the $+3 \cdot 0$ dioptre condition, however, the responses were quite different in size (crossed= $-1 \cdot 5$, uncrossed $=-0.3$ microvolt). Indeed the uncrossed condition evoked almost no response at all. This trend is illustrated well in Fig. 3, which shows the relationship between amplitude and simulated refractive error. The regression line for the crossed condition has a slope of -0.37 microvolts/dioptre, whereas that for the uncrossed condition is -0.65 microvolt/dioptre. The linearity of both regression lines, which comprise the data for the four channels combined, is significant (BMDP program P1R':

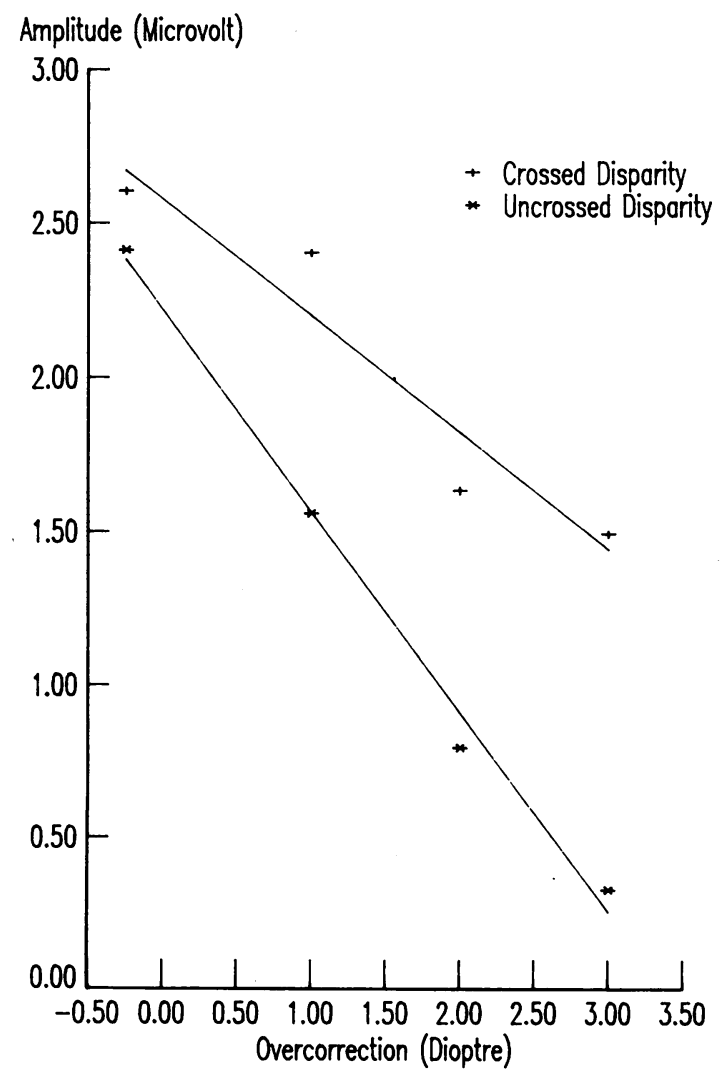

Fig. 3 Amplitude of response is plotted against refractive overcorrection, along with the associated lines of best fit. crossed: $F(1,110)=12.64, p<0.0006$; uncrossed: $F(1,110)=55 \cdot 36, p<0 \cdot 0001)$. The main effect for crossed/uncrossed was significant in the ANOVA of the slopes $(F(1,18)=6 \cdot 59, p<0 \cdot 05)$.

\section{Discussion}

The results of this experiment, both behavioural and electrophysiological, provide strong support for the hypothesis under test. The introduction of a simulated refractive error effectively diminishes the observer's ability to discriminate high-frequency textual detail, which in turn affects stereoscopic resolution. The effect on the ability to resolve changes in uncrossed disparity is significantly greater than the crossed disparity counterpart. The visual system therefore appears to be especially sensitive in the processing of crossed disparity. The result must be qualified by the following comments. Firstly, the experiment was based on the artiicial simulation of a myopic type of refractive error in subjects with essentially normal vision. It will be interesting to repeat the experiment with real myopes to see if progressive refractive correction differentially improves the stereoscopic resolution. Secondly, within the confines of the experimental run it was impracticable to use accommodation blocking drugs. The subjects therefore responded to under-power eyepiece lenses by simply accommodating. Hence, while it would certainly have been desirable to do so, it was not possible to test responses to simulated hypermetropia.

In this experiment the dynamic random dot stimulus comprised a change from zero disparity to disparity and back to zero disparity. At onset this change in disparity was interpreted as a movement towards the viewer in the crossed condition and away in the uncrossed condition. A previous study by one of the authors ${ }^{8}$ has shown that the onset of disparity is responsible for the bulk of the recorded response.

Despite the fact that no subject could perceive the stimulus in the +3.0 dioptre, uncrossed disparity condition, a small but consistent evoked response was recorded, as can be seen in Fig. 2. A previous study has shown that this is very unlikely to be artefactual in origin. ${ }^{9}$ The result is consistent with that of an earlier study ${ }^{10}$ in which a group of nonperceiving subjects produced measurable stereoscopic evoked potentials. The normal response appears to reflect an overlay of processes such as those related to form detection ${ }^{10}$ and to the processing of edge details, ${ }^{9}$ along with more fundamental stereoscopic functions. The conditions imposed on the subjects by the combination of +3.0 dioptre refractive error and uncrossed disparity apparently 
rendered many, but not all, of these processes inoperative.

As an object approaches an observer, the accommodation/convergence reflex assists by keeping the object within the range of focused single vision. A quickly approaching object will generally lead the accommodation and convergence process. Hence the image of the object will be $(a)$ somewhat blurred owing to the delay in the accommodation process, and $(b)$ it will be associated with a crossed disparity due to the trailing convergence process. The findings of this experiment indicate that the visual system is particularly well adapted to cope with this situation.

The result may also have some implications in relation to the properties of vision in squint. A convergent squint results in the observer viewing the world through an inappropriate uncrossed disparity, while a divergent squint results in an inappropriate crossed disparity. The functional consequences of these differences are not clear. Further investigation is certainly warranted.

The authors thank Mrs A Quayle, EEG technician for her significant input to this research project. The research was supported by National Health and Medical Research Council Grants No. 84/0464 and No. 87/0595.

\section{References}

1 Poggio GF, Fischer B. Binocular interaction and depth sensitivity in striate and prestriate cortex of behaving rhesus monkey. J Neurophysiol 1977; 40: 1392-405.

2 Fischer B, Poggio GF. Depth sensitivity of binocular cortical neurons of behaving monkeys. Proc R Soc Lond Biol 1979; 204: 409-14.

3 Manning ML, Finlay DC, Neill RA, Frost BG. Detection threshold differences to crossed and uncrossed disparities. Vision Res in press.

4 Fenelon B, Neill RA, White CT. Evoked potentials to dynamic random dot stereograms in upper, center and lower fields. Doc Ophthalmol 1986; 63: 151-6.

5 Neill RA, Fenelon B. The objective evaluation of stereopsis. Australas Phys Eng Sci Med 1981; 4: 122-5.

6 Neill RA, Kennewell JA. A clinical test for stereopsis. Australas Phys Sci Med 1979; 2: 463-80.

7 Dixon WJ. In: Dixon WJ, ed. BMDP statistical software. Berkeley: UCLA Press, 1985.

8 Neill RA. Physical measurement of human binocular parameters. PhD thesis. University of Newcastle. 1984: 259-65.

9 Neill RA, Fenelon B, Manning ML, Frost BG. Evoked potentials to dynamic random dot stimuli with varying dot density ratios of disparity to background. Doc Ophthalmol 1986; 63: 407-15.

10 Dunlop DB, Dunlop P, Fenelon B, Neill RA. Evoked responses to distinct and nebulous stereoscopic stimuli. Aust J Ophthalmol 1983; 11: 295-301.

Accepted for publication 30 July 1987. 